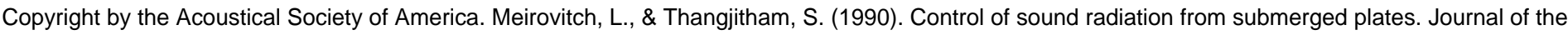
Acoustical Society of America, 88(1), 402-407. doi: 10.1121/1.399916

\title{
Control of sound radiation from submerged plates
}

\author{
Leonard Meirovitcha) and Surot Thangjitham ${ }^{\text {b) }}$ \\ Department of Engineering Science and Mechanics, Virginia Polytechnic Institute and State University, \\ Blacksburg, Virginia 24061
}

(Received 17 January 1989; accepted for publication 6 February 1990)

\begin{abstract}
In many applications, the radiation pressure is an undesirable effect, so that the object is to suppress it. This paper is concerned with radiation pressure suppression through active control. The general idea of active control of a structure is to suppress the vibration by applying suitable feedback control forces, which requires sensors capable of measuring the state of the system and actuators capable of applying control forces. A control technique capable of suppressing the far-field radiation pressure is developed.
\end{abstract}

PACS numbers: 43.30.Jx, 43.20.Rz, 43.40.Vn

\section{INTRODUCTION}

The subject of sound radiation from submerged elastic bodies, and in particular flat plates, is of current research interest in the field of underwater acoustics. Because the plate is in contact with the surrounding fluid, it gives rise to a general class of coupled problems involving "radiation loading." This radiation loading modifies the motion of the submerged plate via the excess acoustic pressure, which in turn depends on the vibration of the plate. As a result, an interaction between the fluid and the plate exists in the form of "feedback coupling."' Such interaction is generally characterized by the scale of motion of the plate surface compared to the acoustic wavelength of the fluid. The interaction becomes significant when the free flexural wave velocity of the plate is less than the velocity of sound in the fluid. ${ }^{2}$

In most situations, the acoustic pressure exerted by a gaseous medium, such as air, on a vibrating plate made of common engineering materials has negligible effect on the dynamic response of the structure. Consequently, the response characteristics of the plate vibrating in the atmosphere are effectively the same as those obtained in vacuo. In contrast, the response characteristics of a vibrating plate submerged in a dense fluid medium can differ considerably from its characteristics in vacuo. This is particularly true for structures having large areas of contact with the surrounding fluid, such as plates and shells.'

Because fluids are generally compressible, a small amount of energy is radiated away from the vibrating plate in the form of sound. ${ }^{2}$ There is a reaction exerted by the fluid on the plate in connection with this sound radiation. This fluid reaction is equivalent to an accession to the inertia of the plate. As a result, it causes an appreciable lowering in its resonant frequencies. In radiating acoustic energy into the fluid, however, a resistive term acting as a damping force on the plate is generated. This damping causes a freely vibrating plate to decay in amplitude. In addition, the fluid serves as a coupling medium by means of which the motion in one mode produces forces acting on another mode, thus causing a

\footnotetext{
a) University Distinguished Professor.

b) Assistant Professor.
}

transfer of energy from one mode to another. ${ }^{1,2}$ Furthermore, it causes the shape of the modes of a freely vibrating plate in contact with fluid to change relative to the corresponding modes in vacuo. From the above discussion, it follows that the problem of determining the structural response to given forces and the problem of determining the acoustic pressure in the surrounding fluid in response to a prescribed velocity distribution at the boundary must be solved simultaneously. More often than not, the acoustic pressure generated by a vibrating plate is an undesirable effect, and the interest lies in suppressing it. To this end, it is common practice to use passive means such as reconfiguring the structure, or using materials with a high damping coefficient. Reconfiguration of the structure may not always be feasible, and passive damping may not always be effective.

The idea of active control of vibration implies the generation of suitable feedback control forces to induce asymptotic stability. ${ }^{3}$ For mere vibration control, the feedback control forces depend on the state of the system, namely, the displacement and velocity. In the case of sound radiation pressure suppression, there is the added task of relating the pressure to the vibration. Active control is carried out by means of sensors and actuators. For practical reasons, the actuators are best located on the structure, and the same can be said about the sensors. After the relation between the structural vibration and the sound radiation pressure has been established, the problem reduces to suppressing the modes of vibration that contribute the most to the radiation pressure. Reference 4 discusses active control of sound radiation pressure in air. This paper is concerned with active control of sound radiation pressure in fluid.

\section{THE PARTIAL DIFFERENTIAL EQUATION OF MOTION}

We are concerned with the problem of controlling a submerged plate, where the plate represents a distributed system governed by the partial differential equation ${ }^{5}$

$$
L w(P, t)+m(P) \ddot{w}(P, t)=f(P, t), \quad P \in \Omega,
$$

in which $w(P, t)$ is the displacement of a typical point $P$ inside the domain $\Omega$ of the system and at time $t, L$ is a homoge- 
neous self-adjoint differential operator of order $2 p, m(P)$ is the mass density, and $f(P, t)$ is a distributed force. The solution $w(P, t)$ of Eq. (1) is subject to the boundary conditions

$$
B_{i} w(P, t)=0, \quad P \in \partial \Omega, i=1,2, \ldots, p,
$$

where $B_{i}$ are boundary differential operators of maximum order $2 p-1$ and $\partial \Omega$ is the boundary of $\Omega$. We assume that the force density can be written in the form

$$
f(P, t)=f_{o}(P, t)+f_{c}(P, t)+f_{d}(P, t)+f_{p}(P, t),
$$

where $f_{o}(P, t)$ is an open-loop control force, $f_{c}(P, t)$ is a closed-loop control force, $f_{d}(P, t)$ is a persistent disturbing force, and $f_{p}(P, t)$ is the pressure force exerted by the fluid on the vibrating plate.

\section{THE MODAL EQUATIONS}

Control of systems described by partial differential equations is not feasible in general, so that we propose to replace the partial differential equation, Eq. (1), by a set of ordinary differential equations. To this end, we consider the open-loop eigenvalue problem

$$
L W(P)=\lambda m(P) W(P), \quad P \in \Omega
$$

where $W(P)$ is subject to the boundary conditions

$$
B_{i} W(P)=0, \quad P \in \partial \Omega, i=1,2, \ldots, p .
$$

Because $L$ is self-adjoint, the solution of the eigenvalue problem consists of a denumerably infinite set of real eigenvalues $\lambda_{r}$ and eigenfunctions $W_{r}(P)$. Assuming that $L$ is positive definite, all $\lambda_{r}$ are positive. We denote them by $\lambda_{r}=\omega_{r}^{2}(r=1,2, \ldots)$, where $\omega_{r}$ are the natural frequencies. Moreover, the eigenfunctions are orthogonal and can be normalized so as to satisfy the orthonormality relations ${ }^{4}$

$$
\begin{aligned}
& \left(W_{s}(P), m(P) W_{r}(P)\right)=\delta_{r s}, \quad r_{0} s=1,2, \ldots, \\
& \left(W_{s}(P), L W_{r}(P)\right)=\omega_{r}^{2} \delta_{r s}, \quad r, s,=1,2, \ldots,
\end{aligned}
$$

where $(g, h)=\int_{\Omega} g h d \Omega$ denotes an inner product and $\delta_{r s}$ is the Kronecker delta.

Using the expansion theorem

$$
w(P, t)=\sum_{r=1}^{\infty} W_{r}(P) \eta_{r}(t),
$$

and following the usual steps, ${ }^{4}$ we obtain the modal equations

$$
\begin{aligned}
\ddot{\eta}_{r}(t)+\omega_{r}^{2} \eta_{r}(t)= & f_{o r}(t)+f_{c r}(t) \\
& +f_{d r}(t)+f_{p r}(t), \quad r=1,2, \ldots,
\end{aligned}
$$

where

$$
\begin{array}{ll}
f_{o r}(t)=\left(W_{r}(P), f_{o}(P, t)\right), & r=1,2, \ldots, \\
f_{c r}(t)=\left(W_{r}(P), f_{c}(P, t)\right), & r=1,2, \ldots, \\
f_{d r}(t)=\left(W_{r}(P), f_{d}(P, t)\right), & r=1,2, \ldots, \\
f_{p r}(t)=\left(W_{r}(P), f_{p}(P, t)\right), & r=1,2, \ldots,
\end{array}
$$

are corresponding modal forces. It is noted that, due to the radiation loading of the fluid, the modal equations, Eqs. (8), are coupled via the modal pressure forces.

We consider the problem of controlling the distributed system by means of a finite number $N$ of discrete actuators acting at the points $P=P_{i}(i=1,2, \ldots, N)$. These actuators are used both for open- and closed-loop control. Discrete actuators can be treated as distributed by writing

$$
\begin{gathered}
f_{o}(P, t)=\sum_{j=1}^{N} F_{o j}(t) \delta\left(P-P_{j}\right), \\
f_{c}(P, t)=\sum_{j=1}^{N} F_{c j}(t) \delta\left(P-P_{j}\right),
\end{gathered}
$$

where $F_{o j}(t)$ and $F_{c j}(t)$ are the open- and closed-loop components of the actuator force $F_{j}(t)$, so that

$$
F_{j}(t)=F_{o j}(t)+F_{c j}(t), \quad j=1,2, \ldots, N .
$$

Inserting Eqs. (10) into Eqs. (9), we obtain

$$
\begin{aligned}
f_{o r}(t) & =\sum_{j=1}^{N} W_{r}\left(P_{j}\right) F_{o j}(t), \\
f_{c r}(t) & =\sum_{j=1}^{N} W_{r}\left(P_{j}\right) F_{c j}(t), \quad r=1,2, \ldots .
\end{aligned}
$$

Control of the entire infinite of modes is not feasible in practice, so that we propose to control a finite number $n$ of modes only. Then, introducing the vectors

$$
\begin{aligned}
\boldsymbol{\eta}_{C}(t) & =\left[\eta_{1}(t) \eta_{2}(t) \cdots \eta_{n}(t)\right]^{T}, \\
\eta_{R}(t) & =\left[\eta_{n+1}(t) \eta_{n+2}(t) \cdots\right]^{T}, \\
\mathbf{f}_{o C}(t) & =\left[f_{o 1}(t) f_{o 2}(t) \cdots f_{o n}(t)\right]^{T}, \\
\mathbf{f}_{o R}(t) & =\left[f_{o(n+1)}(t) f_{o(n+2)}(t) \cdots\right]^{T}, \\
\mathbf{f}_{c C}(t) & =\left[f_{c 1}(t) f_{c 2}(t) \cdots f_{c n}(t)\right]^{T}, \\
\mathbf{f}_{c R}(t) & =\left[f_{c(n+1)}(t) f_{c(n+2)}(t) \cdots\right]^{T}, \\
\mathbf{f}_{d C}(t) & =\left[f_{d 1}(t) f_{d 2}(t) \cdots f_{d n}(t)\right]^{T}, \\
\mathbf{f}_{d R}(t) & =\left[f_{d(n+1)}(t) f_{d(n+2)}(t) \cdots\right]^{T}, \\
\mathbf{f}_{p C}(t) & =\left[f_{p 1}(t) f_{p 2}(t) \cdots f_{p n}(t)\right]^{T}, \\
\mathbf{f}_{p R}(t) & =\left[f_{p(n+1)}(t) f_{p(n+2)}(t) \cdots\right]^{T},
\end{aligned}
$$

as well as the matrices

$$
\begin{aligned}
\Lambda_{C} & =\operatorname{diag}\left(\lambda_{1} \lambda_{1} \cdots \lambda_{n}\right) \\
& =\operatorname{diag}\left(\omega_{1}^{2} \omega_{2}^{2} \cdots \omega_{n}^{2}\right), \\
\Lambda_{R} & =\operatorname{diag}\left(\lambda_{n+1} \lambda_{n+2} \cdots\right) \\
& =\operatorname{diag}\left(\omega_{n+1}^{2} \omega_{n+2}^{2} \cdots\right),
\end{aligned}
$$

Eqs. (8) can be divided into two sets of modal equations, one for the controlled modes, denoted by the subscript $C$, and the other for the uncontrolled (residual) modes, denoted by the subscript $R$. The two sets have the vector form

$$
\begin{aligned}
\ddot{\eta}_{C}(t)+\Lambda_{C} \eta_{C}(t)= & \mathbf{f}_{o C}(t)+\mathbf{f}_{c C}(t) \\
& +\mathbf{f}_{d C}(t)+\mathbf{f}_{p C}(t), \\
\ddot{\eta}_{R}(t)+\Lambda_{R} \eta_{R}(t)= & \mathbf{f}_{o R}(t)+\mathbf{f}_{c R}(t) \\
& +\mathbf{f}_{d R}(t)+\mathbf{f}_{p R}(t) .
\end{aligned}
$$

It should be noted that, due to the modal interaction resulting from fluid loading, these two sets of modal equations are coupled. However, it was shown that, in the case of a simply supported submerged plate, the coupling decreases as the mode number increases. ${ }^{1}$

Introducing Eqs. (12) for the open- and closed-loop control forces into Eqs. (15), the modal equations for the controlled modes and for the residual modes become 


$$
\begin{aligned}
\ddot{\eta}_{C}(t)+\Lambda_{C} \eta_{C}(t)= & B_{C}\left[\mathbf{F}_{o}(t)+\mathbf{F}_{c}(t)\right] \\
& +\mathbf{f}_{d C}(t)+\mathbf{f}_{p C}(t), \\
\ddot{\eta}_{R}(t)+\Lambda_{R} \eta_{R}(t)= & B_{R}\left[\mathbf{F}_{o}(t)+\mathbf{F}_{c}(t)\right] \\
& +\mathbf{f}_{d R}(t)+\mathbf{f}_{p R}(t),
\end{aligned}
$$

where

$$
\begin{aligned}
& \mathbf{F}_{o}(t)=\left[F_{o 1}(t) F_{o 2}(t) \cdots F_{o n}(t)\right]^{T}, \\
& \mathbf{F}_{c}(t)=\left[F_{c 1}(t) F_{c 2}(t) \cdots F_{c n}(t)\right]^{T},
\end{aligned}
$$

are the open- and closed-loop force vectors, respectively, and

$$
\begin{array}{ll}
B_{C}=\left[B_{r i}\right]=\left[W_{r}\left(P_{i}\right)\right], & r=1,2, \ldots, n, \\
& i=1,2, \ldots, N, \\
B_{R}=\left[B_{r i}\right]=\left[W_{r}\left(P_{i}\right)\right], & r=n+1, n+2, n+3, \ldots, \\
& i=1,2, \ldots, N,
\end{array}
$$

are modal participation matrices for the controlled and residual modes, respectively.

\section{CONTROL OF A PERSISTENTLY DISTURBED SYSTEM}

We consider the case in which the persistent disturbance is harmonic with frequency $\omega$. Letting the persistent disturbing force have the form

$$
f_{d}(P, t)=f_{d}(P) e^{i \omega t},
$$

where $f_{d}(P)$ is a distributed force, and using the third of Eqs. $(9)$, we obtain the modal disturbing forces

$$
f_{d r}(t)=\left(W_{r}(P), f_{d}(P, t)\right)=f_{d r} e^{i \omega t}, \quad r=1,2, \ldots,
$$

where

$$
f_{d r}=\int_{\Omega} W_{r}(P) f_{d}(P) d \Omega, \quad r=1,2, \ldots,
$$

are modal disturbing force amplitudes. Equations (20) can be expressed in terms of corresponding vectors for the modal disturbing forces associated with the controlled and residual model as follows:

$$
\begin{aligned}
& \mathbf{f}_{d C}(t)=\mathbf{f}_{d C} e^{i \omega t}, \\
& \mathbf{f}_{d R}(t)=\mathbf{f}_{d R} e^{i \omega t},
\end{aligned}
$$

where the constant vectors $\mathbf{f}_{d C}$ and $\mathbf{f}_{d R}$ are given by

$$
\begin{aligned}
& \mathbf{f}_{d C}=\left[f_{d 1} f_{d 2} \cdots f_{d n}\right]^{T}, \\
& \mathbf{f}_{d R}=\left[f_{d(n+1)} f_{d(n+2)} \cdots\right]^{T},
\end{aligned}
$$

and they define the amplitude of the modal disturbing forces.

\section{EFFECT OF FLUID LOADING ON A SUBMERGED PLATE}

The pressure force exerted by the fluid on the vibrating submerged plate is defined as

$$
f_{p}(P, t)=-p(P, t),
$$

where $p(P, t)$ is the acoustic pressure at the fluid-plate interface. The acoustic pressure field in the fluid is governed by the solution of the three-dimensional wave equation and is related to the flexural displacement component of the plate via the fluid momentum equation at the fluid-plate interface. ${ }^{\prime}$
For a submerged plate subjected to a persistent harmonic disturbance with frequency $\omega$, the acoustic pressure at the fluid-plate interface is given by ${ }^{1}$

$$
\begin{aligned}
p(P, t)= & -\frac{i \rho \omega^{2}}{4 \pi^{2}} \sum_{s=1}^{\infty}\left(\int_{-\infty}^{\infty} \int_{-\infty}^{\infty} \frac{\widetilde{W}_{s}\left(\gamma_{x}, \gamma_{y}\right)}{\sqrt{k^{2}-\gamma_{x}^{2}-\gamma_{y}^{2}}}\right. \\
& \left.\times \exp \left[i\left(\gamma_{x} x+\gamma_{y} y\right)\right] d \gamma_{x} d \gamma_{y}\right) \eta_{s}(t),
\end{aligned}
$$

where $P \equiv(x, y)$ are the in-plane coordinates of the plate, $k$ and $\rho$ are the acoustic wavenumber and density of the fluid, respectively, $\eta_{s}(t)=\eta_{s} \exp (i \omega t)$, and $\widetilde{W}_{s}\left(\gamma_{x}, \gamma_{y}\right)$ is the Fourier integral transform of the eigenfunction $W_{s}(x, y)$ and is defined as

$$
\begin{aligned}
& \tilde{W}_{s}\left(\gamma_{x}, \gamma_{y}\right) \\
& \quad=\int_{-\infty}^{\infty} \int_{-\infty}^{\infty} W_{s}(x, y) \exp \left[-i\left(\gamma_{x} x+\gamma_{y} y\right)\right] d x d y,
\end{aligned}
$$

in which $\gamma_{x}$ and $\gamma_{y}$ are the transform variables.

The modal pressure forces are obtained by calculating the corresponding inner product, which implies substituting Eqs. (24) and (25) into Eq. (9d) and performing the necessary integrations. Consequently, the modal pressure forces become

$$
\begin{aligned}
f_{p r}(t)= & \frac{i \rho \omega^{2}}{4 \pi^{2}} \sum_{s=1}^{\infty}\left(\int_{-\infty}^{\infty} \int_{-\infty}^{\infty} \frac{1}{\sqrt{k^{2}-\gamma_{x}^{2}-\gamma_{y}^{2}}}\right. \\
& \left.\times \widetilde{W}_{s}\left(\gamma_{x}, \gamma_{y}\right) \widetilde{W}_{r}^{*}\left(\gamma_{x}, \gamma_{y}\right) d \gamma_{x} d \gamma_{y}\right) \eta_{s}(t),
\end{aligned}
$$

where $\widetilde{W}_{r}^{*}\left(\gamma_{x}, \gamma_{y}\right)$ is the complex conjugate of $\widetilde{W}_{r}\left(\gamma_{x}, \gamma_{y}\right)$.

Introducing the vectors

$$
\begin{aligned}
& \mathbf{f}_{p}(t)=\left[f_{\rho 1}(t) f_{p 2}(t) \cdots f_{p n}(t) \cdots\right]^{T} \\
& \boldsymbol{\eta}(t)=\left[\eta_{1}(t) \eta_{2}(t) \cdots \eta_{n}(t) \cdots\right]^{T},
\end{aligned}
$$

the modal pressure forces can be written in the convenient form

$$
\mathbf{f}_{p}(t)=C \eta(t),
$$

where $C$ is an $\infty \times \infty$ complex modal coupling impedance matrix defined as

$$
\begin{aligned}
C_{r s}= & \frac{i \rho \omega^{2}}{4 \pi^{2}} \int_{-\infty}^{\infty} \int_{-\infty}^{\infty} \frac{1}{\sqrt{k^{2}-\gamma_{x}^{2}-\gamma_{y}^{2}}} \\
& \times \widetilde{W}_{r}^{*}\left(\gamma_{x}, \gamma_{y}\right) \tilde{W}_{,}\left(\gamma_{x}, \gamma_{y}\right) d \gamma_{x} d \gamma_{y} .
\end{aligned}
$$

When the number of controlled modes is sufficiently large, the coupling between the controlled and uncontrolled modes become negligible, i.e., the off-diagonal entries in the matrix $C$ corresponding to the higher modes tend to zero. This allows the modal pressure forces to be divided into two parts, one associated with the controlled modes and the other with the residual modes, or

$$
\left[\begin{array}{l}
\mathbf{f}_{p C}(t) \\
\mathbf{f}_{p R}(t)
\end{array}\right]=\left[\begin{array}{ll}
C_{C} & 0 \\
0 & C_{R}
\end{array}\right]\left[\begin{array}{l}
\eta_{C}(t) \\
\eta_{R}(t)
\end{array}\right],
$$


where the impedance matrices $C_{C}$ and $C_{R}$ are defined as

$$
\begin{array}{ll}
C_{C}=\left[C_{r s}\right], & r, s,=1,2, \ldots, n, \\
C_{R}=\left[C_{r s}\right], & r, s,=n+1, n+2, \ldots,
\end{array}
$$

where it is noted that $C_{R}$ is a mere diagonal matrix.

Introducing $\mathbf{f}_{p C}(t)$ and $\mathbf{f}_{p R}(t)$ from Eq. (31) into Eqs. (16), the modal equations for the controlled and residual modes become

$$
\begin{aligned}
& \ddot{\eta}_{C}(t)+\left(\Lambda_{C}-C_{C}\right) \eta_{C}(t)=B_{C}\left[\mathbf{F}_{o}(t)+\mathbf{F}_{c}(t)\right]+\mathbf{F}_{d C}(t), \\
& \ddot{\eta}_{R}(t)+\left(\Lambda_{R}-C_{R}\right) \eta_{R}(t)=B_{R}\left[\mathbf{F}_{o}(t)+\mathbf{F}_{c}(t)\right]+\mathbf{F}_{d R}(t) .
\end{aligned}
$$

\section{OPEN-LOOP CONTROL}

We assume that the open-loop control is designed so as to reduce the effect of the disturbing forces on the controlled modes, or

$$
B_{C} \mathbf{F}_{o}(t)+\mathbf{f}_{d C}(t)=\mathbf{0},
$$

which yields the vector of open-loop control forces

$$
\mathbf{F}_{o}(t)=-B_{C}^{\dagger} \mathbf{f}_{d C}(t),
$$

where

$$
B_{C}^{\dagger}=\left(B_{C}^{T} B_{C}\right)^{-1} B_{C}^{T}
$$

is the pseudo-inverse of $B_{C}$.

Inserting Eq. (35) into Eq. (33a), we obtain

$$
\ddot{\eta}_{C}(t)+\left(\Lambda_{C}-C_{C}\right) \eta_{C}(t)=B_{C} \mathbf{F}_{c}(t)+D \mathbf{f}_{d C}(t),
$$

where

$$
D=I-B_{C} \boldsymbol{B}_{C}^{\dagger} .
$$

From Eqs. (37) and (38), we conclude that the effect of the persistent disturbances remains, albeit in reduced form. The extent to which this effect is still present depends on how close $D$ is to a null matrix, which in turn depends on how close $B_{C}^{\dagger}$ is to an actual inverse $B_{C}^{-1}$. Clearly, the effect diminishes as $B_{C}$ approaches a square matrix and disappears completely when $B_{C}$ is square, which happens when the number of actuators is equal to the number of controlled modes, $N=n$.

\section{CLOSED-LOOP CONTROL STATE EQUATIONS}

In designing closed-loop control, it is often convenient to cast the equations of motion in state form, particularly if the object is optimal control. Hence, let us introduce the modal state vector associated with the controlled modes

$$
\mathbf{w}_{C}(t)=\left[\boldsymbol{\eta}_{C}^{T}(t) \dot{\boldsymbol{\eta}}_{C}^{T}(t)\right]^{T} \text {, as well as the coefficient matrices }
$$

$$
\begin{gathered}
A_{C}=\left[\begin{array}{ccc}
0 & I \\
\hdashline-\left(\Lambda_{C}-C_{C}\right) & 0
\end{array}\right], \\
B_{C}^{\prime}=\left[\begin{array}{c}
0 \\
-- \\
B_{C}
\end{array}\right], D^{\prime}=\left[\begin{array}{c}
0 \\
-- \\
D
\end{array}\right] .
\end{gathered}
$$

Then, adjoining the identity $\dot{\eta}_{C}(t) \equiv \dot{\eta}_{C}(t)$, Eq. (37) can be rewritten in the form

$$
\dot{\mathbf{w}}_{C}(t)=A_{C} \mathbf{w}_{C}(t)+B_{C}^{\prime} \mathbf{F}_{c}(t)+D^{\prime} \mathbf{f}_{d C}(t) .
$$

We consider an optimal control $\mathbf{F}_{c}(t)$ that minimizes the performance index

$$
\begin{aligned}
J= & \frac{1}{2} \mathbf{w}_{C}^{T}\left(t_{f}\right) H \mathbf{w}_{C}\left(t_{f}\right) \\
& \left.+\frac{1}{2} \int_{t_{i}}^{t_{f}} \mathbf{w}_{C}^{T} Q \mathbf{w}_{C}+\mathbf{F}_{c}^{T} R \mathbf{F}_{c}\right) d t,
\end{aligned}
$$

where $t_{i}$ and $t_{f}$ denote the initial and final time, respectively, and $H, Q$, and $R$ are coefficient matrices. It can be shown that minimization of $J$ yields the optimal control law ${ }^{3}$

$\mathbf{F}_{c}(t)=-R^{-1}\left(B_{C}^{\prime}\right)^{T} P(t) \mathbf{w}_{C}(t)=G(t) \mathbf{w}_{C}(t)$,

where $G(t)$ is a control gain matrix, in which $P(t)$ is a $2 n \times 2 n$ matrix satisfying the matrix Riccati equation

$$
\begin{aligned}
\dot{P}(t)=- & P(t) A_{C}-A_{C}^{T} P(t)-Q \\
& +P(t) B_{C}^{\prime} R^{-1}\left(B_{C}^{\prime}\right)^{T} P(t), \quad P\left(t_{f}\right)=H .
\end{aligned}
$$

For sufficiently large $t_{f}$, the Riccati matrix $P(t)$ tends to a constant value. In this case, $P$ is determined by solving the steady-state Riccati equation, which is obtained from Eq. (43) by letting $\dot{P}=0$. The resulting nonlinear matrix algebraic equation can be solved by means of Potter's method, ${ }^{3}$ which amounts to solving an eigenvalue problem of twice the order of the Riccati equation. We are concerned with the case in which the Riccati matrix is constant, so that the control gain matrix $G$ is constant. The closed-loop state equations are obtained by inserting Eq. (42) into Eq. (40). The result is

$$
\dot{\mathbf{w}}_{C}(t)=\left(A_{C}-B_{C}^{\prime} G\right) \mathbf{w}_{C}(t)+D^{\prime} \mathbf{f}_{d C}(t) .
$$

\section{SOLUTION OF THE CLOSED-LOOP EQUATIONS}

We propose to solve Eq. (44) by expressing the solution as a linear combination of the closed-loop eigenvectors multiplied by some time-dependent functions. To this end, we must first solve the closed-loop eigenvalue problem

$$
A_{\mathrm{CL}} U=U \Lambda_{\mathrm{CL}}, \quad V^{T} A_{\mathrm{CL}}=\Lambda_{\mathrm{CL}} V^{T},
$$

- where

$$
A_{\mathrm{CL}}=A_{C}-B_{C}^{\prime} G
$$

is the closed-loop coefficient matrix, $U$ and $V$ are matrices of the closed-loop right and left eigenvectors, respectively, and $\Lambda_{\mathrm{CL}}$ is the diagonal matrix of closed-loop eigenvalues. The eigenvectors are normalized so as to satisfy the biothornormality relations

$$
V^{T} U=I, \quad V^{T} A_{\mathrm{CL}} U=\Lambda_{\mathrm{CL}} .
$$

Then, introducting the linear transformation

$$
\mathbf{w}_{C}(t)=U \mathbf{u}_{C}(t)
$$

into Eq. (44), multiplying on the left by $V^{T}$, considering Eqs. (47) and recalling Eq. (22a), we obtain

$$
\dot{\mathbf{u}}_{C}(t)=\Lambda_{C L} \mathbf{u}_{C}(t)+V^{T} D^{\prime} \mathbf{f}_{d C} e^{i \omega t},
$$


which represents a set of independent equations.

The solution of Eq. (49) has the form

$\mathbf{u}_{C}(t)=e^{\Lambda_{C L} t^{t}} \mathbf{u}_{C}(0)+\left(i \omega I-\Lambda_{\mathrm{CL}}\right)^{-1} V^{T} D^{\prime} \mathbf{f}_{d C} e^{i \omega t}$,

where, from Eqs. (47) and (48),

$$
\mathbf{u}_{C}(0)=V^{T} \mathbf{w}_{C}(0) \text {, }
$$

in which $w_{C}(0)$ is the initial modal state vector. Finally, inserting Eqs. (48) and (51) into Eq. (50), we obtain the solution of the closed-loop state equations

$$
\begin{aligned}
\mathbf{w}_{C}(t)= & U e^{\Lambda_{C L}{ }^{t} V^{T} \mathbf{w}_{C}(0)} \\
& +U\left(i \omega I-\Lambda_{\mathrm{CL}}\right)^{-1} V^{T} D^{\prime} \mathbf{f}_{d C} e^{i \omega t} .
\end{aligned}
$$

\section{EFFECT OF THE RESIDUAL MODES}

From Eq. (52), we observe that the feedback control causes the effect of any transient excitation on the controlled modes to decay with time. Moreover, the effect of the persistent excitation is further reduced due to the phase shift. Equation (52) does not provide a complete picture of the system behavior, but only of the behavior of the controlled modes. The question remains as to how the control affects the uncontrolled residual modes. To answer this question, we use Eq. (33b) and write the modal equations for the residual modes:

$$
\ddot{\eta}_{R}(t)+\left(\Lambda_{R}-C_{R}\right) \eta_{R}(t)=\mathbf{N}_{R}(t),
$$

where, using Eqs. (22b), (35), (42), and (52),

$$
\begin{aligned}
\mathbf{N}_{R}(t)= & B_{R}\left[\mathbf{F}_{o}(t)+\mathbf{F}_{c}(t)\right]+\mathbf{f}_{d R}(t) \\
= & -B_{R} G U \mathrm{e}^{\Lambda_{\mathrm{CL} .}{ }^{t}} V^{T} \mathbf{w}_{C}(0) \\
& +\left\{\mathbf{f}_{d R}-B_{R}\left[B_{C}^{\dagger}+G U(i \omega I\right.\right. \\
& \left.\left.\left.-\Lambda_{\mathrm{CL}}\right)^{-1} V^{T} D^{\prime}\right] \mathbf{f}_{d C}\right\} e^{i \omega t} .
\end{aligned}
$$

Clearly, the first term on the right side of Eq. (54) represents a transient excitation and the second term a steady-state excitation. Equation (53) represents an independent set of equations. The steady-state solution is simply

$$
\begin{aligned}
\eta_{R s \mathrm{~s}}(t)=- & \left(\omega^{2} I-\Lambda_{R}+C_{R}\right)^{-1}\left\{\mathbf{f}_{d R}-B_{R}\left[B_{C}^{\dagger}\right.\right. \\
& \left.\left.+G U\left(i \omega I-\Lambda_{\mathrm{CL}}\right)^{-1} V^{T} D^{\prime}\right] \mathbf{f}_{d C}\right\} e^{i \omega t},
\end{aligned}
$$

in which the subscript $s s$ stands for steady state and does not identify a mode.

\section{THE FAR-FIELD RADIATION PRESSURE}

The object of the control is to suppress the sound radiation pressure generated by the vibrating structure. Hence, to verify the effectiveness of the control, it is necessary to relate the radiation pressure to the vibration. For structures of complex configuration, this relation is not within the state of the art. A problem of considerable interest, and one permitting a solution, is that of rectangular plates. For rectangular planar radiators, the far-field sound pressure is given by Rayleigh's formula:

$$
\begin{aligned}
p(R, \theta, \phi, t)= & \frac{\rho e^{i k R}}{2 \pi R} \int_{-a}^{a} \int_{-b}^{b} \ddot{w}(x, y, t) \\
& \times \exp [-i k \sin \theta(x \cos \phi \\
& +y \sin \phi)] d x d y,
\end{aligned}
$$

where $\ddot{w}(x, y, t)$ is the acceleration of a point $x, y$ on the radiator, $\rho$ and $k$ are the density and wavenumber of the baffle, respectively, $R, \theta$, and $\phi$ are spherical coordinates, and $2 a$ and $2 b$ are the dimensions of the plate.

\section{CONTROL OF A RECTANGULAR PLATE}

We propose to suppress the acoustic pressure radiated from a vibrating simply supported uniform rectangular plate. The dimensions of the plate are $2 a$ and $2 b$ in the $x$ and $y$ directions, and the thickness is equal to $h$. The differential operator appearing in Eq. (1) has the expression ${ }^{5}$

$$
L=D \nabla^{4}=D\left(\frac{\partial^{4}}{\partial x^{4}}+2 \frac{\partial^{4}}{\partial x^{2} \partial y^{2}}+\frac{\partial^{4}}{\partial y^{4}}\right),
$$

where $D=E h^{3} / 12\left(1-v^{2}\right)$ is the plate's stiffness, in which $E$ is the modulus of elasticity, and $v$ is Poisson's ratio. We note that $p=2$. Moreover, if the origin of the $x$ and $y$ axes is at the center of the plate, the boundary operators are

$$
\begin{aligned}
& B_{1}=1, \text { and } B_{2}=\frac{\partial^{2}}{\partial x^{2}}, \text { along } x= \pm a, \\
& B_{1}=1 \text {, and } B_{2}=\frac{\partial^{2}}{\partial y^{2}}, \quad \text { along } y= \pm b .
\end{aligned}
$$

The natural frequencies have the values ${ }^{4}$

$$
\omega_{r s}=\frac{\pi^{2}}{4}\left[\left(\frac{r}{a}\right)^{2}+\left(\frac{s}{b}\right)^{2}\right] \sqrt{\frac{D}{m}}, \quad r, s=1,2, \ldots,
$$

where $m$ is the mass per unit area of plate, and the associated eigenfunctions are

$$
\begin{aligned}
W_{r s}(x, y)= & \frac{1}{\sqrt{m a b}} \sin \frac{r \pi(x+a)}{2 a} \\
& \times \sin \frac{s \pi(y+b)}{2 b}, \quad r, s,=1,2, \ldots,
\end{aligned}
$$

where the eigenfunctions have been normalized so that $\int_{-a}^{a} \int_{-b}^{b} m W_{r s}^{2} d x d y=1$.

From Eqs. (52) and (55), we can write the steady-state portion of the controlled and residual modal displacement vectors in the general form

$$
\boldsymbol{\eta}_{C . s s}(t)=\mathbf{H}_{C}(\omega) e^{i \omega t}, \quad \eta_{R s s}(t)=\mathbf{H}_{R}(\omega) e^{i \omega t},
$$

where $\mathbf{H}_{C}(\omega)$ and $\mathbf{H}_{R}(\omega)$ play the role of frequency response vectors. Then, denoting by $\mathbf{W}_{C}(x, y)$ and $\mathbf{W}_{R}(x, y)$ the vectors of corresponding eigenfunctions for the controlled and residual modes, respectively, and using Eq. (7), the steady-state response can be written as follows:

$$
\begin{aligned}
w_{s s}(x, y, t)= & {\left[\mathbf{W}_{C}^{T}(x, y) \mathbf{H}_{C}(\omega)\right.} \\
& \left.+\mathbf{W}_{R}^{T}(x, y) \mathbf{H}_{R}(\omega)\right] e^{i \omega t} .
\end{aligned}
$$

In the case of steady-state harmonic response, it is convenient to rewrite formula (56) in terms of frequency response functions. To this end, we identify the contribution of a single mode, namely, $W_{r s}(x, y)$ to the steady-state response given by Eq. (62) and write this contribution in the form

$$
w_{r s}(x, y, t)=W_{r s}(x, y, \omega) e^{i \omega t}, \quad r, s=1,2, \ldots,
$$

where 


$$
\begin{aligned}
W_{r s}(x, y, \omega)= & H_{r s}(\omega) \sin \frac{r \pi(x+a)}{2 a} \\
& \times \sin \frac{s \pi(y+b)}{2 b}, \quad r, s,=1,2, \ldots,
\end{aligned}
$$

in which $H_{r s}(\omega)$ is the frequency response associated with $W_{r s}(x, y)$. Similarly, we can write the contribution of the mode $W_{r s}(x, y)$ to the far-field radiation pressure as

$$
p_{r s}(R, \theta, \phi, t)=p_{r s}(R, \theta, \phi, \omega) e^{i \omega t},
$$

where, using Eqs. (56), (63), and (65),

$$
\begin{aligned}
p_{r s}(R, \theta, \phi, \omega)= & -\frac{\omega^{2} \rho e^{i k R}}{2 \pi R} \int_{-a}^{a} \int_{-b}^{b} W_{r s}(x, y, \omega) \\
& \times \exp [-i k \sin \theta(x \cos \phi \\
& +y \sin \phi)] d x d y .
\end{aligned}
$$

At this point, it will prove convenient to introduce the notation

$$
\sin \theta \cos \phi=\alpha, \quad \sin \theta \sin \phi=\beta,
$$

as well as

$$
r \pi / 2 a=k_{r}, \quad s \pi / 2 b=k_{s} .
$$

Then, inserting Eq. (64) into Eq. (66) and evaluating the integral, we obtain

$$
\begin{aligned}
p_{r s}(R, \theta, \phi, \omega)= & -\frac{\omega^{2} \rho e^{i k R}}{2 \pi R} \int_{-a}^{a} \int_{-b}^{b} H_{r s}(\omega) \sin k_{r}(x+a) \\
& \times \sin k_{s}(y+b) e^{-i k(\alpha s+\beta y)} d x d y \\
= & -\frac{\rho e^{i k R}}{2 \pi R} \omega^{2} H_{r s}(\omega) \frac{k_{r}}{k_{r}^{2}-k^{2} \alpha^{2} k_{s}^{2}-k^{2} \beta^{2}} \\
& \times\left(e^{i r \pi} e^{-i k \alpha a}-e^{i k \alpha a}\right)\left(e^{i s \pi} e^{-i k \beta b}-e^{i k \beta b}\right) \\
= & \left(\rho e^{i k R} / 2 \pi R\right) P_{r s}(\theta, \phi, \omega),
\end{aligned}
$$

where $P_{r s}(\theta, \phi, \omega)$ is a measure of the far-field pressure frequency response associated with the mode $W_{r s}(x, y)$.

The total far-field radiation pressure at any given point $(R, \theta, \phi)$ is obtained by summing up the contributions from all the vibrating modes, or

$$
\begin{aligned}
p(R, \theta, \phi, \omega) & =\frac{\rho e^{i k R}}{2 \pi R} \sum_{r=1}^{\infty} \sum_{s=1}^{\infty} P_{r s}(\theta, \phi, \omega) \\
& =\frac{\rho e^{i k R}}{2 \pi R} P(\theta, \phi, \omega),
\end{aligned}
$$

where $P(\theta, \phi, \omega)$ is proportional to the far-field pressure frequency response, and it includes contributions from all modes.

\section{CONCLUSIONS}

This paper develops a technique for the active control of sound radiation from a vibrating plate submerged in fluid. The plate is subjected to a persistent excitation in the form of a harmonic force. The modes of vibration of the "radiation loaded" plate are divided into controlled and uncontrolled, or residual modes. A relation between the modes of vibration and the far-field radiation pressure is given. This relation can be used to select the modes to be controlled.

'M. C. Junger and D. Feit, Sound, Structures and Their Interaction (MIT, Cambridge, MA, 1982), 2nd ed.

${ }^{2}$ L. Cremer, M. Heck, and E. E. Unger, Structure-Borne Sound (Springer, New York, 1973).

${ }^{3}$ L. Meirovitch, Dynamics and Control of Structures (Wiley-Interscience, New York, 1990).

${ }^{4} \mathrm{~L}$. Meirovitch and S. Thangjitham, "Active Control of Sound Radiation Pressure," Journal of Vibration and Acoustics (to be published).

${ }^{5}$ L. Meirovitch, Analytical Methods in Vibrations (Macmillan, New York, 1967). 